\title{
Serine/threonine protein phosphatases in DNA damage response
}

\author{
LIU Bo \& XU XingZhi* \\ Beijing Key Laboratory of DNA Damage Response, College of Life Sciences, Capital Normal University, Beijing 100048, China
}

Received April 29, 2011; accepted June 30, 2011

\begin{abstract}
DNA damage response (DDR) is among the most important of the mechanisms that maintain genome stability which, when destabilized, predisposes organs to cancer. Reversible phosphorylation mediated by protein kinases and protein phosphatases regulates most, if not all, cellular activities, including DDR. Protein kinase inhibitors have become the main focus of targeted therapy and anticancer drug development. However, our limited knowledge of protein phosphatase function is compromising our capacity to develop therapeutic agents against phosphatases. In this review, we summarize the roles of serine/threonine protein phosphatases involved in DDR and propose that in situ dephosphorylation of phosphoproteins by protein phosphatases, instead of proteasome-mediated degradation of phosphoproteins, is mainly employed by cells.
\end{abstract}

reversible protein phosphorylation, protein phosphatase, DNA damage, cancer

Citation: $\quad$ Liu B, Xu X Z. Serine/threonine protein phosphatases in DNA damage response. Chinese Sci Bull, 2011, 56: 3122-3131, doi: 10.1007/s11434$011-4679-5$

The human genome is being constantly attacked by various DNA damaging agents. According to the source of the toxic agents, DNA damage can be categorized into 2 groups, endogenous (spontaneous) and exogenous (environmental) $[1,2]$. Endogenous DNA damage occurs at a high frequency within normal cells, mainly caused by by-products of metabolic and biochemical reactions, such as reactive oxygen species (ROS), reactive chemicals (e.g. aldehydes and $S$ adenosylmethionine). In most cases, these endogenous toxic agents result in the modification or hydrolysis of the base components of DNA, such as through oxidation, alkylation, deamination, depurination and depyrimidination. Mismatch is another kind of endogenous DNA damage occasionally introduced during DNA replication [3-5].

DNA damage caused by exogenous agents, such as ionizing radiation (IR) (ultraviolet (UV), X-rays, and $\gamma$-rays) and radiomimetic drugs is much more catastrophic than that caused by endogenous agents. UV radiation has deleterious effects in all living organisms, prokaryotic bacteria and eukaryotes, lower and higher plants, and animals including humans. UV radiation can be classified into 3 categories

*Corresponding author (email: xingzhi_xu@mail.cnu.edu.cn) according to its wavelength or frequency: long wave UVA (315-400 nm), medium wave UVB (280-315 nm), and short wave UVC (100-280 nm) [6]. Most UV radiation $(98.7 \%)$ is blocked by the Earth's ozone layer when penetrating through the atmosphere, including most of UVB (95\%) and all of UVC. Although UVA and UVB induce similar mutations in human skin cells [7], UVB is the primary cause of UV radiation damage, as it is directly absorbed by cellular DNA and an increasing amount reaches the Earth because of substantial damage to the protective ozone layer [6,8]. UVA causes less direct DNA damage because it is not absorbed by cellular DNA. Gamma rays and radiomimetic drugs are the most dangerous types of DNA damaging agents, which can cause lethal lesions, such as DNA double-strand breaks (DSBs), and they have been widely used to kill cancerous cells in cancer therapy. Radiomimetic drugs, such as camptothecin (CPT) and etoposide are both effective anticancer drugs, which specifically bind and inhibit the enzyme activity of topoisomerase I and II respectively, which unwind DNA during replication or transcription [9]. Adriamycin and bleomycin interact with DNA by intercalation and inhibit the macromolecular biosynthesis $[10,11]$. 


\section{DNA damage response}

One single cell may incur tens of thousands of DNA lesions daily. Fortunately, mammalian cells have developed evolutionarily conserved mechanisms to cope with genotoxic threats, which have been termed as the DNA damage response (DDR) (Figure 1). Upon DNA damage, cells detect the damaged DNA and relay the signal downstream to activate the cell cycle checkpoints to halt the cell cycle progression, allowing time for repairing the damage, or they tolerate the damage and continue replication, which may eventually cause mutations, or lead to apoptosis if the damage is too severe to be repaired [12-14]. Several conserved repair pathways responsible for eliminating specific types of lesions have been identified in mammalian cells. These include direct repair, base excision repair (BER), nucleotide excision repair (NER), mismatch repair (MMR), homologous recombination (HR) repair, nonhomologous end joining (NHEJ) repair, fanconi anemia (FA) repair and translesion DNA synthesis (TLS) [2,15-18]. There is also considerable redundancy among these different pathways. A class of enzyme known as alkyltransferases can repair DNA base damage induced by alkylating agents by directly removing the alkyl group. Both BER and NER repair single-strand DNA damage. HR and NHEJ are mainly responsible for DSB repair [19-21].

DDR involves 3 main groups of evolutionarily conserved

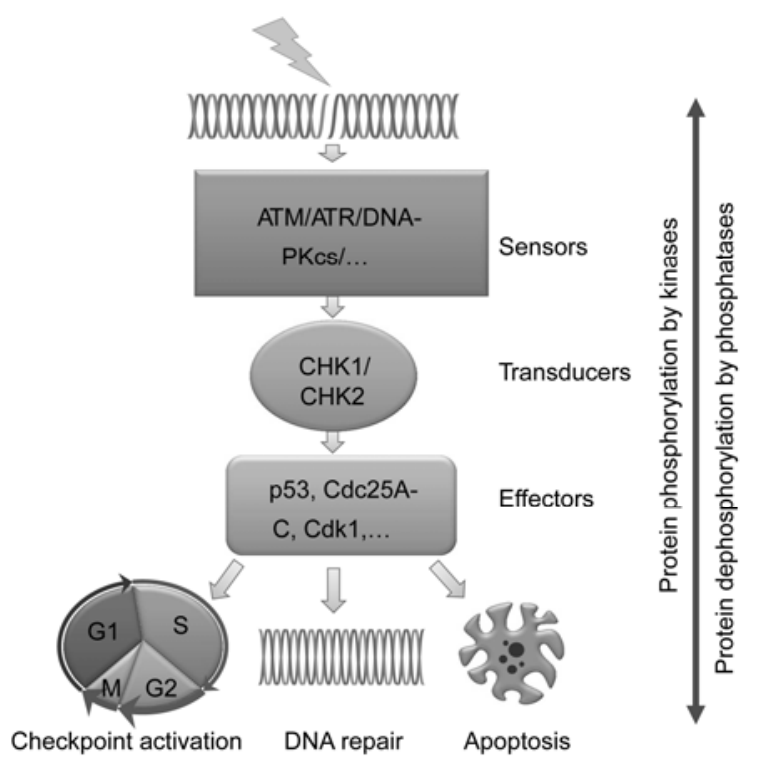

Figure 1 A simplified schematic diagram of the DNA damage response (DDR). Members of the PIKK family of serine-threonine kinases ATM, ATR, and DNA-PKcs, and their interacting partners detect the damaged DNA and initiate signals to the checkpoint kinases CHK1 and CHK2. Both CHK1 and CHK2 phosphorylate various substrates, activating cell cycle checkpoints to allow time for DNA repair, fixing damaged DNA, and promoting apoptosis when DNA damage is too severe to be repaired. Reversible protein phosphorylation mediated by kinases and phosphatases takes part in almost every step of the DDR. proteins that transduce the DNA damage signals and determine the cell fate (Figure 1). The first group comprises the DNA damage sensor proteins, such as ATM (ataxia telangiectasia mutated protein), ATR (ataxia telangiectasia and Rad3-related protein), and DNA-PKcs (DNA-dependent protein kinase catalytic subunit), which belong to the phosphoinositide 3-kinase-related kinase (PIKK) family. It also includes their physical and functional partners, including MRE11-RAD50NBS1, PARP-1 (poly(adenosine diphosphate ribose) polymerase 1), TIP60 (60 kD Tat-interactive protein), MDC1 (mediator of DNA damage checkpoint 1), ATRIP (ATRinteracting protein), Claspin, RAD17-RFC (replication factor C), Ku70/80, and Artemis. The second group comprises the transducer proteins, including checkpoint kinases CHK1 and CHK2 that relay the signals to the downstream effectors, which are the third group [22,23] and control the cell cycle progression, remodeling the chromatin or repairing the damage $[13,24,25]$. The DDR pathway is a cascade of reversal phosphorylation events of the sensors, transducers and effectors. Large-scale proteomic analysis of proteins phosphorylated in response to DNA damage (caused by ionizing radiation) on consensus sites recognized by ATM and ATR have identified more than 900 regulated phosphorylation sites encompassing over 700 proteins, most of which are involved in DDR [26].

For example, upon DSB formation, chromatin remodeling and protein and DNA modification result in dissociation of the sensor protein ATM, changing it from a dimer into a monomer and causing autophosphorylation on multiple residues required for its activation [27]. The activated ATM phosphorylates H2AX on serine (Ser) 139, located at the C-terminal, to form $\gamma \mathrm{H} 2 \mathrm{AX}$. MDC1 directly binds to $\gamma \mathrm{H} 2 \mathrm{AX}$ through its $\mathrm{C}$-terminal tandem BRCT domains (the C-terminal portion of the $B R C A-1$ gene) [28]. At the same time, MDC1 facilitates the transfer of the DNA damage signal through ATM-dependent phosphorylation of downstream targets on S/T-Q residues, generating a landing platform for RNF8 [29,30]. Furthermore, ATM activates CHK2 by phosphorylation of $\mathrm{T} 68$, and both kinases phosphorylate various substrates, such as p53 and CDC25, to activate the cell cycle checkpoints [31,32].

\section{Protein phosphatases}

Reversible protein phosphorylation, mediated by protein kinases and protein phosphatases, occurs on about one-third of all proteins in human cells, and controls almost every aspect of cellular activities, including DDR [33]. Disturbed protein phosphorylation has been identified in many human diseases, including cancer. Proteins, such as enzymes and receptors, are activated or inactivated by reversible phosphorylation, which results in a conformational change in the proteins structure.

Phosphorylation occurs predominantly on serine (Ser), 
threonine (Thr) and tyrosine (Tyr) residues, with each accounting for approximately $86.4 \%, 11.8 \%$ and $1.8 \%$, respectively, of the human phosphoproteome [34]. In addition, basic amino acid residues, such as histidine, arginine or lysine, in prokaryotic proteins can also be modified via phosphorylation [35]. The human genome encodes 518 protein kinases, 428 of which phosphorylate Ser or Thr residues, and the remaining 90 kinases belong to the Tyr kinase family [36-38]. By contrast, there are only about 147 protein phosphatase catalytic subunits identified in the human genome, with 107 serving as Thr phosphatases or dual-specificity phosphatases [38]. Surprisingly, the human genome only encodes $40 \mathrm{Ser} / \mathrm{Thr}$ phosphatases, whereas more than $98 \%$ of the phosphorylation occurs on Ser/Thr residues.

Phosphatases can be classified into 3 groups according to their sequence identity, structure, substrate specificity and catalytic mechanisms, namely Ser/Thr protein phosphatases, Tyr protein phophatases, dual specificity protein phosphatases, and the FCP/SCP/HAD family. The FCP/SCP/HAD family uses an Asp-based catalysis mechanism with a DXDXT/V catalytic motif in which the first aspartate serves as a phosphoryl acceptor during the substrate dephosphorylation [39].

Ser/Thr protein phosphatases comprise the PPP (phosphoprotein phosphatase) family and the PPM (protein phosphatase, $\mathrm{Mg}^{2+}$ or $\mathrm{Mn}^{2+}$ dependent family, also PP2C family) family and are responsible for dephosphorylation of phosphoserines or phosphothreonines [40]. The catalytic subunit of the PPP family members itself does not have any enzymatic activity. It forms heterodimeric or heterotrimeric holoenzymatic complexes with regulatory/scaffolding subunits. These holoenzymes thus gain catalytic activity, specific subcellular localization, and specific substrate recognition. The PPP family is further divided into 3 subfamilies: PP1, PP2A and PP2B. PP4 and PP6 are closely related to PP2A, while PP7 was grouped into the PP2B subfamily because of its requirement for $\mathrm{Ca}^{2+}$ [41]. Most members of this family have roles in DDR [42]. The PPM members are catalytically $\mathrm{Mg}^{2+}$ or $\mathrm{Mn}^{2+}$ dependent, sharing structural similarity, however, there is no sequence homology with the PPP members at their active sites. The human genome encodes 18 PPM members [43]. The catalytic subunits of this family exhibit enzymatic activity without the requirement of additional regulatory subunits, however, their interacting partners may play a role in determining their subcellular localization and modulate their enzymatic activity. PPM1D or WIP1 (wild type p53-inducible phosphatase 1) is a significant member of this family involved in DDR (Table 1).

To date, 107 protein Tyr phosphatases (PTPs) have been identified in the human genome, 106 of which have mouse orthologs, and only 81 of these are catalytically active. Compared with about 90 catalytically active Tyr kinases, it is reasonable to assume that the PTPs have comparable substrate specificities. PTPs have been found to play critical roles in human diseases, such as diabetes, Sézary syndrome, and Noonan syndrome, and at least 30 PTPs have been implicated in tumor growth [38]. PTPs are becoming a promising drug target in drug development [44].

We will summarize Ser/Thr protein phosphatases involved in DDR (Table 1) in the following sections.

\subsection{PP1}

Protein phosphatase 1 (PP1) is widely expressed in mammalian cells. It includes 3 highly-related isoforms, PP1 $\alpha$, PP1 $\beta$, and PP1 $\gamma$, encoded by separated genes [45]. These isoforms have more than $89 \%$ identity in amino acid sequence. PP1 $\gamma$ has two alternative splicing variants, PP1 $\gamma 1$ and PP1 $\gamma 2$ [46]. Localization patterns of PP1 isoforms are dynamic in a cell cycle-dependent manner. PP1 $\alpha$ localizes to the nucleus during the interphase, while PP1 $\beta$ and PP1 $\gamma$ additionally accumulate in the nucleoli $[47,48]$. PP1 has been reported to interact with various subunits through its $\mathrm{N}$ terminus. Sequence analysis of all the interacting partners has revealed that most of these proteins contain a consensus PP1-binding motif: $(\mathrm{R} / \mathrm{K}) \times 1(\mathrm{~V} / \mathrm{I}) \times 2(\mathrm{~F} / \mathrm{W})$, where $\times 1$ may be absent or any residue apart from the large hydrophobic residues, and $\times 2$ denotes any amino acid except large hydrophobic residues, phosphoserine and probably aspartic

Table 1 Ser/Thr protein phosphatases and their substrates in DDR

\begin{tabular}{|c|c|c|c|c|c|c|}
\hline Sub-family & \multicolumn{2}{|c|}{ Phosphatases } & Catalytic subunits & Regulatory subunits & Involved in DDR & Identified substrates in DDR \\
\hline \multirow[t]{6}{*}{ PPP } & \multicolumn{2}{|c|}{ PP1 } & $\alpha, \beta, \gamma 1, \gamma 2$ & $>90$ & yes & $\begin{array}{l}\text { CDC25, CHK1, BRCA1, p53, } \\
\text { KAP1, etc. }\end{array}$ \\
\hline & PP2A-like & PP2A & $\alpha, \beta$ & B subunits & yes & $\begin{array}{l}\gamma \mathrm{H} 2 \mathrm{AX}, \mathrm{p} 53, \mathrm{DNA}-\mathrm{PKcs}, \mathrm{Chk} 2 \text {, } \\
\text { etc. }\end{array}$ \\
\hline & & PP4 & PPP4C & $\begin{array}{l}\text { PP4R1, PP4R2, PP4R3 } \alpha, \\
\text { PP4R3 } \beta, \text { PP4R4 }\end{array}$ & yes & $\gamma \mathrm{H} 2 \mathrm{AX}, \mathrm{RPA} 2$ \\
\hline & & PP6 & PPP6C & PP6R1, PP6R2, PP6R3 & yes & $\gamma \mathrm{H} 2 \mathrm{AX}$ \\
\hline & \multicolumn{2}{|l|}{ PP5 } & PP5 & - & yes & - \\
\hline & \multicolumn{2}{|l|}{ PP7 } & PP7 & - & unknown & - \\
\hline $\mathrm{PPM} / \mathrm{PP} 2 \mathrm{C}$ & \multicolumn{2}{|c|}{$\begin{array}{l}\text { PPM1A, B, etc. } \\
\text { (18 members) }\end{array}$} & PPM1A, B, etc. & - & PPM1D & $\begin{array}{l}\text { ATM, ATR, CHK1, CHK2, } \\
\text { p53, } \gamma \text { H2AX, etc. }\end{array}$ \\
\hline
\end{tabular}


acid [49,50]. More than 180 conserved mammalian proteins have been identified as potential binding partners of PP1. PP1 exists in as many as 650 distinct complexes, which may contribute to its subcellular localizations, substrates specificity, and account for the roles of PP1 in the regulation of an enormous variety of cellular functions [50,51]. One prerequisite for activation of $\mathrm{PP} 1$ is the removal of the inhibitory phosphothreonine residue at the PP1 C-terminus, which is phosphorylated by cyclin-dependent kinases (CDKs) in a cell cycle-dependent manner [52].

The roles of PP1 in DDR have been reported in both DNA damage signaling and DNA repair. Upon DNA damage, PP1 is activated after ionizing radiation by removing the inhibitory phosphorylation sites in an ATM dependent manner [53]. PP1 also regulates the ATM activation by its chromatin-targeting subunit, Repo-Man [54]. Studies in Xenopus egg extracts demonstrate that Repo-Man interacts with ATM and PP1 through distinct domains, leading to PP1-dependent regulation of ATM phosphorylation and activation. Overexpression of the wild-type Repo-Man, but not the PP1-binding deficient mutant, attenuates the DNA damage-induced ATM activation [53,55,56]. The mechanisms underlying PP1 participation in DNA damage during interphase and mitosis have been elucidated by identification of several other PP1 substrates, such as CDC25, CHK1, BRCA1 and p53 [57-62]. CDC25, a crucial regulator of the G2/M transition, is a highly conserved dual-specific phosphatase and a key target of the checkpoint machinery that ensures genetic stability.

Dis2, the Schizosaccharomyces pombe homolog of PP1, is required for dephosphorylation and deactivation of CHK1 and recovery from DNA damage-induced G2 checkpoint arrest $[59,60]$. BRCA1 is also identified as a PP1 substrate during DNA damage recovery [61,63]. PP1 $\alpha$ interacts with BRCA1 via its PP1-binding motif (898KVTF901) and mediates dephosphorylation of BRCA1 at several sites, which were previously phosphorylated by ATM, ATR, or CHK2 upon DNA damage. Mutation in the PP1-binding motif in BRCA1 impaired the DNA damage-induced Rad51 foci formation, and compromised its function in the $\operatorname{HR}[55,61$, 63]. Moreover, low levels of BRCA1 may be related to the variable levels of $\mathrm{PP} 1 \alpha$ and $\beta$ in primary sporadic human breast tumors, indicating an important role of PP1 during the development of breast cancer, and may result from an aberration in its phosphorylation status [62]. p53 is another substrate of PP1. As a multifunction factor, the roles and phosphorylation status of p53 have been well established during the past decades [64]. Upon DNA stress, such as $\gamma$-irradiation or UV irradiation, p53 is phosphorylated at Ser15 and Ser20 by ATM/CHK2 and ATR/CHK1 [65-68]. PP1 dephosphorylates UV-induced p53 phosphorylation at Ser15, and this can be disturbed by okadaic acid (OA) and GADD34 (growth arrest and DNA damage 34). GADD34, one of the PP1 binding partners, inhibits PP1 binding with p53, interfers with the dephosphorylation of p53 and sus- tains the amount of phospho-p53 after UV-treatment [69]. New data suggest that PP1 may be involved in the regulation of cross talk between different post-translational modifications in DDR, e.g., KAP1 (Krüppel-associated box associated protein 1) phosphorylation and sumoylation. KAP1 provides a prosurvival advantage by contributing to transcriptional repression of the DNA damage response genes p21, Bax, noxa and Puma. KAP1 phosphorylation antagonizes its sumoylation and keeps it inactive. PP1 $\alpha$ is essential to establish the minimal level of Ser-824 phosphorylation required for KAP1's corepressor function in unstressed cells. By contrast, PP1 $\beta$ is recruited to KAP1 post Dox-treatment, and its stimulatory effect on sumoylation is expanded from KAP1 to RanGAP1. These findings provide novel mechanistic insights into how PP1 impacts DDR, influencing not only KAP1 Ser-824 dephosphorylation, but also its sumoylation and targeted gene expression [2].

\subsection{PP2A}

PP2A is a major Ser/Thr phosphatase with distinct roles in regulation of cell cycle progression, cell growth and development, cytoskeleton dynamics, and cell mobility [70]. Two isoforms of PP2A are encoded by 2 distinct and unlinked genes, PP2AC $\alpha$ and PP2AC $\beta$. These 2 isoforms share $97 \%$ identity in primary amino acid sequences, there is no antibody available to date that can distinguish them [70]. They are expressed in all tissues and cell types and distributed in the cytosol, nucleus, and chromatins. Knockout of Ppp2c $\alpha$ in mice leads to death at embryonic day 5.5. It has been assumed that they are functionally redundant. However, it would be interesting to see the phenotypes of $P p p 2 c \beta$ knockout mice and if these two isoforms can rescue each other in vivo [71].

Active forms of PP2A exist as heterotrimeric complexes, each of which consists of a catalytic subunit $\mathrm{C}$, a structural subunit A and a regulatory subunit B [70]. The structural subunit A interacts with the catalytic $\mathrm{C}$ subunit by its carboxyl terminus, and forms a horseshoe-shaped scaffold. There are two A isoforms encoded by two alternative genes, $P R 65 \alpha$ and $P R 65 \beta$, with $87 \%$ similarity in their primary amino acid sequences [72]. The heterodimer AC remains inactive without the addition of the regulatory $\mathrm{B}$ subunits. To date, 15 genes have been identified in the human genome. These genes encode at least 26 different alternatively spliced variants representing the B subunits of the PP2A holoenzyme. These B subunits have been divided into 4 families based on sequence homology, namely, B (B55 or PR55), B' (B56 or PR61), B"(PR48/59/72/130), and B'" (PR93/110) families. It is believed that PP2A achieves regulatory flexibility and substrate specificity via the specific association of the core dimer with one of the regulatory B subunits [73].

Involvement of PP2A in DNA damage signaling, DNA repair and apoptosis has been established. Upon DNA damage, PP2A is activated, partially regulated by methyla- 
tion on the catalytic domain [74]. PP2A regulates the DNA damage-induced G1/S checkpoint, and several checkpoint proteins, including $\mathrm{Rb}$ (retinoblastoma), p107, and p53, which are PP2A substrates [70,75]. UV-induced dephosphorylation of $\mathrm{Rb}$ and $\mathrm{p} 107$ by PP2A facilitates their interaction with E2F to arrest cells in the G1 phase, inhibiting cell proliferation. Furthermore, PP2A participates in the net accumulation of un(der)-phosphorylated $\mathrm{Rb}$ in the soluble fraction following DNA damage in the S phase. PP2A dephosphorylates p53 at Ser37 and Thr55 [76]. Dephosphorylation of p53 Ser37 downregulates transcriptional activity of p53 in response to DNA damage. Thr55 dephosphorylation stabilizes p53 in response to DNA damage, and a prerequisite of this process is the ATM-dependent phosphorylation of p53 at Ser15, which enhances the interaction between PP2A regulatory subunit B56( $\gamma)$,(p53) [77].

PP2A has an essential role in the activation of the IRinduced G2/M checkpoint. Expression of the oncogenic inhibitor of the PP2A catalytic subunits and HOX11 in T-cell lines stops the G2 arrest induced by $\gamma$ irradiation. Furthermore, inhibition of PP2A by inhibitors or siRNA abrogates the IR-induced activation of ATR and CHK1, as well as phosphorylation of Cdc2-Tyr15, and attenuates the IR-induced G2/M arrest [78]. Similarly, expression of an N-terminally truncated form of the B56 subunit in 3T3 cells also abrogates radiation-induced $\mathrm{G} 2$ arrest. PP2A might also be involved in the deactivation of PLK1 by dephosphorylation upon mitotic DNA damage [79]. PP2A/B56 $\delta$ is also involved in the regulation of the activity of CDC25 at the G2/M transition and recovery from DNA damage, by dephosphorylating Cdc25 at a site (T138) whose phosphorylation is required for 14-3-3 release [80].

Various checkpoint signaling factors are substrates of PP2A. For example, PP2A may dephosphorylate DNA-PK protein kinase and thus regulate its kinase activity [81]. Phosphorylation-induced inhibition of the DNA-PK kinase activity is restored by the addition of a purified PP1 or PP2A catalytic subunit, and this reactivation is obstructed by microcystin, a protein phosphatase inhibitor [81]. PP2A counteracts ATM- and ATR-mediated DNA damage checkpoints in Xenopus egg extracts independent of Cdk2- and Cdc7-mediated checkpoints, and it is required for the activation of the ATR-CHK1 pathway in response to $\mathrm{Vpr}$ (viral protein R) [81,82]. Upon IR-induced DSBs or other DNA lesions, CHK2 is phosphorylated at Thr68 and activated in an ATM-dependent manner, then it phosphorylates various downstream proteins, including B subunits of PP2A, increasing its phosphatase activity [82]. PP2A in turn interacts with and dephosphorylates Thr68-CHK2 to turn off the DNA damage signal when the damage has been repaired, forming a negative regulation feedback loop.

\subsection{PP4}

PP4 is closely related to PP2A, exhibiting phosphatase ac- tivity in a heterodimeric or heterotrimeric holoenzyme [83, 84]. PP4 has been known to be a ubiquitous Ser/Thr phosphatase in several species for more than a decade. Mammalian PP4c share $65 \%$ amino acid identity with PP2c $\alpha$ and $\mathrm{PP} 2 \mathrm{c} \beta$. Five regulatory subunits have been identified, PP4R1, PP4R2, PP4R3 $\alpha$, PP4R3 $\beta$ and PP4R4, forming 4 potential holoenzymatic complexes, PP4c-PP4R1, PP4c-PP4R4, PP4cPP4R2-PP4R3 $\alpha$, and P4c-PP4R2-PP4R3 $\beta[83,84]$. PP4 has roles in several cellular processes, including organelle assembly, centrosome maturation, spliceosome assembly, regulation of histone acetylation and chromatin remodeling [85-87]. Its roles in DDR have been appreciated recently. Gingras and colleagues identified several novel, evolutionarily conserved PP4C-containing complexes involved in cisplatin sensitivity [88]. Deletion of any of the PP4 subunit orthologs in Saccharomyces cerevisiae elicited cisplatin hypersensitivity [89]. Furthermore, human PP4R3 complemented yeast psy2 deletion, and Drosophila melanogaster lacking functional PP4R3 (falafel) exhibited cisplatin hypersensitivity, suggesting a highly conserved role for PP4 in DNA damage repair. Finally, PP4R3 was found to, at least in part, target PP4c to the DNA damage repair machinery via an interaction with Rad53 (hCHK2) [42,88,90]. Investigations in budding yeasts demonstrated that phosphatase Pph3-containing complex facilitated DNA damage checkpoint recovery by regulating the phosphorylation status of $\mathrm{H} 2 \mathrm{~A}$. In addition, Pph3 forms a complex with PsyII (Pph3-PsyII) that specifically binds and dephosphorylates Rad53 during replication stress recovery, this is required to restart stalled replication forks [84,91].

Studies in mammalian cells have revealed that PP4C, PP4R2, and PP4R3 $\beta$ holoenzymatic complexes specifically dephosphorylate ATR-mediated $\gamma \mathrm{H} 2 \mathrm{AX}$ generated during DNA replication $[83,92]$. The PP4C-PP4R2 complex regulated the RPA2 phosphorylation level to regulate in turn the HR repair [93]. Overexpression of PP4 has been found in human breast tumors and lung tumors, and inhibition of its expression sensitizes cancer cells to cisplatin treatment [94]. Overexpression of PP4 and other protein phosphatases in tumors may confer resistance to chemotherapeutic drug-induced DNA damage because DNA damage-induced $\gamma \mathrm{H} 2 \mathrm{AX}$ may be dephosphorylated regardless of repair. This would allow cancer cells to continue proliferating in the presence of damaged DNA. These studies have established that PP4 is involved in DDR and could be a target for drug development and cancer therapy.

\subsection{PP6}

PP6 is the mammalian homolog of yeast Sit4, which is highly related in sequence to the PP2A-like Ser/Thr phosphatases. Phenotypes induced by inhibition of Sit 4 could be rescued by ectopic expression of mammalian PP6 [95]. The phosphatase activity of Sit4 depends on its interacting partners, Sit4-associated proteins (SAP) [96]. Three regulatory 
subunits containing SAP domains, which were renamed as PP6R1, PP6R2 and PP6R3, respectively, have been identified in mammals and shown to coprecipitate with PP6c, but not with PP4 or PP2A [97,98]. All three SAP subunits can individually associate with PP6c, but exhibit nonoverlapping substrate specificities. DNA-PK interacts with PP6C, and its regulatory subunits PP6R1, PP6R2 and PP6R3. Silencing of PP6 leads to sensitivity to IR and delays release from the G2/M checkpoint [99]. Furthermore, silencing of PP6c or PP6R1 resulted in sustained phosphorylation of $\gamma \mathrm{H} 2 \mathrm{AX}$ after IR, while neither of the phosphorylation states of DNA-PK or ATM was compromised [99]. This demonstrated that neither DNA-PK nor ATM is a substrate of PP6c, DNA-PK may instead recruit PP6 to sites of DNA damage to dephosphorylate $\gamma \mathrm{H} 2 \mathrm{AX}$, remove IR-induced foci, and release from the G2/M checkpoint in vivo [99]. This appears contradictory to the findings of other reports [99-101], in which inhibition of PP6c or PP6R1 expression by siRNA impairs DNA-PK activation in response to IR, though all reports demonstrated that preventing PP6c expression sensitizes tumor cells to IR. Our laboratory further demonstrated that depletion of PP6c or PP6R2, but not PP6R1, compromised repair of CPT-induced DSBs or I-SceI-induced DSB, suggesting that DNA lesions induced by different agents may require different PP6 holoenzymes [102].

Our laboratory also examined expression status of PP6c and its regulatory subunits in breast tumor samples derived from a cohort of approximately 200 breast cancer patients [102]. It was unexpected to find that expression of PP6c, PP6R2, and PP6R3 was lowered in breast cancer samples in comparison with benign breast lesions, suggesting that PP6 may have a protective role during breast tumorigenesis.

\section{$2.5 \quad$ PP5}

PP5 is a unique phosphatase of the PPP family, not only in terms of its structure, but also its phosphatase activity. PP5 has a peptidyl-prolyl cis-trans-isomerase-like (PPIase-like) domain and 3 consecutive tetratricopeptide repeat (TPR) domains responsible for protein interaction, neither of which have been identified in other protein phosphatases [103]. Its phosphatase domain resides in the C-terminus and shares about $48 \%$ sequence similarity to other phosphatases, such as PP1, PP2A and PP2B. PP5 exhibits low phosphatase activity under normal conditions, which may be a consequence of the inhibitory interaction between its C-terminus region (responsible for the phosphatase activity) and its $\mathrm{N}$-terminus TPR region [103]. PP5 plays roles in proliferation, migration, differentiation, electrolyte balance, apoptosis, survival, and also DDR [103,104]. PP5 is required for the activation of both ATM-mediated and ATR-mediated DNA damage checkpoints through enhanced direct interaction in response to genotoxic stresses [105-107]. PP5 interacts with ATM depending on DNA damage, and inhibition of PP5 expression attenuated ATM activation. The mouse model lacking PP5 does not arrest the ATM-mediated cell cycle [108]. PP5 is also required for the activation of ATR and phosphorylation of Rad17 and CHK1 after UV or hydroxyurea treatment [107]. While no phosphorylation residues have been identified that can be reversed by PP5, the available data suggest that PP5 may regulate the phosphorylation status of 53BP1 in response to radiomimetic agents, such as neocarzinostatin (NCS) [109]. Overexpression of PP5 was found in human breast cancer, indicating that elevated PP5 protein levels may promote tumor progression [110].

\subsection{PPM1D}

PPM1D/WIP1 was originally identified as a wild-type p53 induced protein phosphatase in response to IR [111]. Subsequent studies have revealed that a wide range of damage agents, such as UV, anisomycin, $\mathrm{H}_{2} \mathrm{O}_{2}$ and MMS (methyl methanesulfonate) also induced its expression [111,112]. It is a monomeric Ser/Thr phosphatase with monogenic activity. Its phosphatase activity requires the presence of $\mathrm{Mg}^{2+}$ or $\mathrm{Mn}^{2+}$, and it is relatively insensitive to OA treatment [113]. Overexpression of PPM1D has been reported in multiple human cancers, including neuroblastomas, pancreatic adenocarcinomas, and medulloblastomas, as well as ovarian and gastric carcinomas [114-117]. Its oncogenic properties have been elucidated in several tumor suppressor pathways [118]. Overexpression of PPM1D in cells removes the IRor UV-induced intra-S and G2-M cell cycle checkpoints, whereas repression of PPM1D expression prolongs these checkpoints [119,120]. Identification of its substrates, which share a $\mathrm{p}(\mathrm{S} / \mathrm{T}) \mathrm{Q}$ motif, has also revealed the critical function of PPM1D as a regulator of DDR [113,121]. Upon UV-irradiation, p53 is phosphorylated by the stress-responsive kinase p38 mitogen-activated protein kinase, at Ser33 and Ser46, which enhances the activity of p53 and ultimately results in cell cycle arrest or apoptosis [122]. The activated p53 then induces the expression of PPM1D, which in turn attenuates the phosphorylation of p53 as well as the conserved active site of $\mathrm{p} 38$, to suppress its transcriptional activity and UV-induced apoptosis. Furthermore, PPM1D facilitates inactivation of the BER by dephosphorylating and deactivating the crucial regulator $\mathrm{UNG} 2$, which is a nuclear isoform of uracil-DNA glycosylase activated upon UV damage [123]. Thus, p-38 MAPK-p53-PPM1D forms a negative feedback loop in the UV-induced DNA damage response. The ATR-CHK1-p53 pathway also occurs upon UV-induced DDR. Upon UV damage, ATR phosphorylates and activates CHK1 at Ser345, as well as p53 at Ser15. At the same time, the activated CHK1 also phosphorylates p53 at Ser20 to stop the interaction between p53 and MDM2, which is an E3 ligase and expressed in a p53 dependent manner, thus stabilizing p53 after DNA damage [124]. In response to DSBs, ATM turns into an active form via auto- 
phosphorylation and monomerization [125], and then phosphorylates multiple downstream effectors, including CHK2T68, p53-Ser15 [125,126]. The activated CHK2 phosphorylates p53-Ser20 and MDM2, leading to the stabilization of p53 [127]. Activated p53 induces the expression of PPM1D, which in turn dephosphorylates and inactivates the upstream proteins, ultimately diminishing the DNA damage signal [121]. $\gamma \mathrm{H} 2 \mathrm{AX}$ is another new substrate reported to be dephosphorylated by PPM1D during checkpoint recovery, and the activity of PPM1D must be tightly regulated to ensure proper damage repair $[128,129]$. These studies indicate that PPM1D is an important negative regulator of DDR.

\section{Phosphatases for $\gamma \mathrm{H} 2 \mathrm{AX}$}

H2AX is rapidly phosphorylated by ATM/ATR/DNA-PK on Ser139 $(\gamma \mathrm{H} 2 \mathrm{AX})$ surrounding sites of DNA damage [130], which serve as a platform for recruitment and enrichment of DDR factors [131,132]. $\gamma \mathrm{H} 2 \mathrm{AX}$ is a marker for DSBs. It has been puzzling when and how $\gamma \mathrm{H} 2 \mathrm{AX}$ is removed. Thus far, several protein phosphatases have been reported to dephosphorylate $\gamma \mathrm{H} 2 \mathrm{AX}$, these include PP2A, PP4C, PP6C, PPM1D and PPM1G [83,92,99,128,133,134]. We and our collaborators have shown that PP4C forms complexes with PP4R2, and PP4R3 $\beta$, and specifically dephosphorylates ATR-mediated $\gamma \mathrm{H} 2 \mathrm{AX}$ within the mono-nucleosomes generated during DNA replication. PP2A is mainly responsible for $\gamma \mathrm{H} 2 \mathrm{AX}$ in response to exogenous DNA damage [83]. We and our collaborators have also revealed that $\gamma \mathrm{H} 2 \mathrm{AX}$ is dephosphorylated by PP6 both in vitro and in vivo [99,102]. Silencing of either PP6c or PP6R1 led to sustained $\gamma \mathrm{H} 2 \mathrm{AX}$ levels after IR, whereas inhibition of PP6c or PP6R2 expression, but not PP6R1 expression, resulted in sustained $\gamma \mathrm{H} 2 \mathrm{AX}$ levels after CPT treatment [102]. It is very interesting that $\gamma \mathrm{H} 2 \mathrm{AX}$ generated under different circumstances is dephosphorylated by different phosphatases, and if by the same phosphatase, the requirement of regulatory/scaffolding subunits is different. These studies confirm that different regulatory/scaffolding subunits may be a key determinant of phosphatase-substrate specificity.

\section{Perspective}

OA-mediated inhibition effects have been attributed to PP2A phosphatase. In fact, OA also blocks phosphatase activity of PP4 and PP6. Therefore, it is necessary to re-examine if PP4 or PP6 participates in or regulates these "assumed" PP2A-mediated cellular activities reported in the literature.

Proteasome-mediated degradation of phosphoproteins has been validated in a significant number of phospho-targets, however, degradation and protein resynthesis are extremely costly in terms of adenosine triphosphate consumption. Given the smartness of both normal cells and cancer cells, the advantage of using this costly approach to deactivate a phosphoprotein is not clear. Though protein phosphatemediated in situ dephosphorylation of phosphoproteins has been only identified to date in significantly fewer phospho- targets, we hypothesize that in situ dephosphorylation by protein phosphatases is a major pathway that deactivates phosphoproteins.

One third of eukaryotic proteins are phosphorylated and more than $98 \%$ of phosphorylation events occur on Ser/Thr residues. The human genome only encodes about 140 catalytic subunits of protein phosphatases, about 40 of which are Ser/Thr phosphatases. Therefore, it would be very delicate and complex for a phosphatase to exhibit its specificity for substrate recognition and recruitment. As discussed above, complex formation of a holoenzyme plays an important role in determining its substrate specificity. It is a challenging and important task to determine physiological substrates and their corresponding specific holo-phosphatases in the near future.

Reversible protein phosphorylation plays a critical role in tumorigenesis. Protein kinases have become the second most important target in the development of anticancer drugs, and it is logical to expect that protein phosphatases are also potential anticancer drug targets. Indeed, a number of natural compounds have been identified to target the catalytic subunit and block its phosphatase activity, however, these compounds have either not been tested in a clinical trial or they failed in the early stage of a trial because of their side effects. These side effects result from the fact that a phosphatase catalytic subunit may have many targets involved in many molecular pathways. To achieve pathwayor substrate-specific blockage, rational design of small molecules targeting the catalytic-substrate interaction or the regulatory subunit-substrate interaction is important.

This work was supported by the startup fund from Capital Normal University, the National Natural Science Foundation of China (30570371, 90608014, 30711120570 and 31071190), the Program for New Century Excellent Talents in University (NCET-06-0187), Beijing Natural Science Foundation Program and Scientific Research Key Program of Beijing Municipal Commission of Education (KZ200810028014), the National Basic Research Program of China (2010CB911904), and Funding Project for Academic Human Resources Development in Institutions of Higher Learning under the Jurisdiction of Beijing Municipality (PHR20110508) to XU XingZhi.

1 Bartsch H, Risch A, Schmezer P, et al. DNA damage from endogenous processes, workshop: 16-17 September 1996, German Cancer Research Centre Heidelberg, Germany. J Cancer Res Clin Oncol, 1997, 123: 232-234

2 Lindahl T, Barnes D E. Repair of endogenous DNA damage. Cold Spring Harb Symp Quant Biol, 2000, 65: 127-133

3 Valko M, Rhodes C J, Moncol J, et al. Free radicals, metals and antioxidants in oxidative stress-induced cancer. Chem Biol Interact, 2006, 160: $1-40$

4 Blasiak J, Arabski M, Krupa R, et al. Basal, oxidative and alkylative 
DNA damage, DNA repair efficacy and mutagen sensitivity in breast cancer. Mutat Res, 2004, 554: 139-148

5 Friedberg E C, McDaniel L D, Schultz R A. The role of endogenous and exogenous DNA damage and mutagenesis. Curr Opin Genet Dev, 2004, 14: 5-10

6 Sinha R P, Hader D P. UV-induced DNA damage and repair: A review. Photochem Photobiol Sci, 2002, 1: 225-236

7 Kappes U P, Luo D, Potter M, et al. Short- and long-wave UV light (UVB and UVA) induce similar mutations in human skin cells. J Invest Dermatol, 2006, 126: 667-675

8 Madronich S, McKenzie R L, Bjorn L O, et al. Changes in biologically active ultraviolet radiation reaching the Earth's surface. J Photochem Photobiol B, 1998, 46: 5-19

9 D'Arpa P, Liu L F. Topoisomerase-targeting antitumor drugs. Biochim Biophys Acta, 1989, 989: 163-177

10 Binaschi M, Capranico G, De Isabella P, et al. Comparison of DNA cleavage induced by etoposide and doxorubicin in two human smallcell lung cancer lines with different sensitivities to topoisomerase II inhibitors. Int J Cancer, 1990, 45: 347-352

11 Azarova A M, Lyu Y L, Lin C P, et al. Roles of DNA topoisomerase II isozymes in chemotherapy and secondary malignancies. Proc Natl Acad Sci USA, 2007, 104: 11014-11019

12 Clemenson C, Marsolier-Kergoat M C. DNA damage checkpoint inactivation: Adaptation and recovery. DNA Repair (Amst), 2009, 8: 1101-1109

13 Kurz E U, Lees-Miller S P. DNA damage-induced activation of ATM and ATM-dependent signaling pathways. DNA Repair (Amst), 2004, 3: 889-900

$14 \mathrm{Su} \mathrm{T}$ T. Cellular responses to DNA damage: One signal, multiple choices. Annu Rev Genet, 2006, 40: 187-208

15 de Gruijl F R, van Kranen H J, Mullenders L H. UV-induced DNA damage, repair, mutations and oncogenic pathways in skin cancer. $\mathrm{J}$ Photochem Photobiol B, 2001, 63: 19-27

16 Helleday T, Petermann E, Lundin C, et al. DNA repair pathways as targets for cancer therapy. Nat Rev Cancer, 2008, 8: 193-204

17 Liu T, Ghosal G, Yuan J, et al. FAN1 acts with FANCI-FANCD2 to promote DNA interstrand cross-link repair. Science, 2010, 329: 693-696

18 Li X, Heyer W D. Homologous recombination in DNA repair and DNA damage tolerance. Cell Res, 2008, 18: 99-113

19 Wang Q, Gao F, Wang T, et al. A nonhomologous end-joining pathway is required for protein phosphatase $2 \mathrm{~A}$ promotion of DNA doublestrand break repair. Neoplasia, 2009, 11: 1012-1021

20 Schwartz M, Zlotorynski E, Goldberg M, et al. Homologous recombination and nonhomologous end-joining repair pathways regulate fragile site stability. Genes Dev, 2005, 19: 2715-2726

21 Mladenov E, Kalev P, Anachkova B. The complexity of doublestrand break ends is a factor in the repair pathway choice. Radiat Res, 2009, 171: 397-404

22 Harper J W, Elledge S J. The DNA damage response: Ten years after. Mol Cell, 2007, 28: 739-745

23 Ciccia A, Elledge S J. The DNA damage response: Making it safe to play with knives. Mol Cell, 2010, 40: 179-204

24 Kanaar R, Wyman C. DNA repair by the MRN complex: Break it to make it. Cell, 2008, 135: 14-16

25 Czornak K, Chughtai S, Chrzanowska K H. Mystery of DNA repair: The role of the MRN complex and ATM kinase in DNA damage repair. J Appl Genet, 2008, 49: 383-396

26 Matsuoka S, Ballif B A, Smogorzewska A, et al. ATM and ATR substrate analysis reveals extensive protein networks responsive to DNA damage. Science, 2007, 316: 1160-1166

27 Bakkenist C J, Kastan M B. DNA damage activates ATM through intermolecular autophosphorylation and dimer dissociation. Nature, 2003, 421: 499-506

28 Stucki M, Clapperton J A, Mohammad D, et al. MDC1 directly binds phosphorylated histone $\mathrm{H} 2 \mathrm{AX}$ to regulate cellular responses to DNA double-strand breaks. Cell, 2005, 123: 1213-1226

29 Marteijn J A, Bekker-Jensen S, Mailand N, et al. Nucleotide excision repair-induced $\mathrm{H} 2 \mathrm{~A}$ ubiquitination is dependent on MDC1 and RNF8 and reveals a universal DNA damage response. J Cell Biol, 2009, 186: 835-847

30 Shao G, Lilli D R, Patterson-Fortin J, et al. The Rap80-BRCC36 deubiquitinating enzyme complex antagonizes RNF8-Ubc13-dependent ubiquitination events at DNA double strand breaks. Proc Natl Acad Sci USA, 2009, 106: 3166-3171

$31 \mathrm{Xu}$ X, Stern D F. NFBD1/MDC1 regulates ionizing radiation-induced focus formation by DNA checkpoint signaling and repair factors. FASEB J, 2003, 17: 1842-1848

32 Ahn J Y, Schwarz J K, Piwnica-Worms H, et al. Threonine 68 phosphorylation by Ataxia telangiectasia mutated is required for efficient activation of Chk2 in response to ionizing radiation. Cancer Res, 2000, 60: 5934-5936

33 Cohen P. The role of protein phosphorylation in human health and disease. The Sir Hans Krebs Medal Lecture. Eur J Biochem, 2001, 268: 5001-5010

34 Olsen J V, Blagoev B, Gnad F, et al. Global, in vivo, and site-specific phosphorylation dynamics in signaling networks. Cell, 2006, 127: 635-648

35 Cozzone A J. Post-translational modification of proteins by reversible phosphorylation in prokaryotes. Biochimie, 1998, 80: 43-48

36 Manning G, Whyte D B, Martinez R, et al. The protein kinase complement of the human genome. Science, 2002, 298: 1912-1934

37 Manning G, Plowman G D, Hunter T, et al. Evolution of protein kinase signaling from yeast to man. Trends Biochem Sci, 2002, 27: 514-520

38 Alonso A, Sasin J, Bottini N, et al. Protein tyrosine phosphatases in the human genome. Cell, 2004, 117: 699-711

39 Moorhead G B, Trinkle-Mulcahy L, Ulke-Lemee A. Emerging roles of nuclear protein phosphatases. Nat Rev Mol Cell Biol, 2007, 8: 234-244

40 Gallego M, Virshup D M. Protein serine/threonine phosphatases: Life, death, and sleeping. Curr Opin Cell Biol, 2005, 17: 197-202

41 Andreeva A V, Kutuzov M A. PPP family of protein Ser/Thr phosphatases: Two distinct branches? Mol Biol Evol, 2001, 18: 448-452

42 Heideker J, Lis E T, Romesberg F E. Phosphatases, DNA damage checkpoints and checkpoint deactivation. Cell Cycle, 2007, 6: 3058-3064

43 Wehenkel A, Bellinzoni M, Schaeffer F, et al. Structural and binding studies of the three-metal center in two mycobacterial PPM Ser/Thr protein phosphatases. J Mol Biol, 2007, 374: 890-898

44 Cohen P. Protein kinases - the major drug targets of the twenty-first century? Nat Rev Drug Discov, 2002, 1: 309-315

45 Song Q, Khanna K K, Lu H, et al. Cloning and characterization of a human protein phosphatase 1-encoding cDNA. Gene, 1993, 129: 291295

46 Fardilha M, Wu W, Sa R, et al. Alternatively spliced protein variants as potential therapeutic targets for male infertility and contraception. Ann N Y Acad Sci, 2004, 1030: 468-478

47 Barker H M, Craig S P, Spurr N K, et al. Sequence of human protein serine/threonine phosphatase 1 gamma and localization of the gene (PPP1CC) encoding it to chromosome bands 12q24.1-q24.2. Biochim Biophys Acta, 1993, 1178: 228-233

48 Trinkle-Mulcahy L, Sleeman J E, Lamond A I. Dynamic targeting of protein phosphatase 1 within the nuclei of living mammalian cells. $\mathbf{J}$ Cell Sci, 2001, 114: 4219-4228

49 Godet A N, Guergnon J, Maire V, et al. The combinatorial PP1binding consensus Motif $(\mathrm{R} / \mathrm{K}) \mathrm{x}((0,1)) \mathrm{V} / \mathrm{IxFxx}(\mathrm{R} / \mathrm{K}) \mathrm{x}(\mathrm{R} / \mathrm{K})$ is a new apoptotic signature. PLoS One, 2010, 5: e9981

50 Hendrickx A, Beullens M, Ceulemans H, et al. Docking motif-guided mapping of the interactome of protein phosphatase-1. Chem Biol, 2009, 16: 365-371

51 Janssens V, Longin S, Goris J. PP2A holoenzyme assembly: In cauda venenum (the sting is in the tail). Trends Biochem Sci, 2008, 33: 113-121

52 Dohadwala M, da Cruze Silva E F, Hall F L, et al. Phosphorylation and inactivation of protein phosphatase 1 by cyclin-dependent kinases. Proc Natl Acad Sci USA, 1994, 91: 6408-6412

53 Guo C Y, Brautigan D L, Larner J M. Ionizing radiation activates nuclear protein phosphatase-1 by ATM-dependent dephosphorylation. J 
Biol Chem, 2002, 277: 41756-41761

54 Peng A, Lewellyn A L, Schiemann W P, et al. Repo-man controls a protein phosphatase 1-dependent threshold for DNA damage checkpoint activation. Curr Biol, 2010, 20: 387-396

55 Tang X, Hui Z G, Cui X L, et al. A novel ATM-dependent pathway regulates protein phosphatase 1 in response to DNA damage. Mol Cell Biol, 2008, 28: 2559-2566

56 Peng A, Lewellyn A L, Schiemann W P, et al. Repo-Man controls a protein phosphatase 1-dependent threshold for DNA damage checkpoint activation. Curr Biol, 2010, 20: 387-396

57 Margolis S S, Perry J A, Weitzel D H, et al. A role for PP1 in the $\mathrm{Cdc} 2 /$ Cyclin B-mediated positive feedback activation of Cdc25. Mol Biol Cell, 2006, 17: 1779-1789

58 Margolis S S, Walsh S, Weiser D C, et al. PP1 control of M phase entry exerted through 14-3-3-regulated Cdc25 dephosphorylation. EMBO J, 2003, 22: 5734-5745

59 den Elzen N R, Kosoy A, Christopoulos H, et al. Resisting arrest: Recovery from checkpoint arrest through dephosphorylation of Chk1 by PP1. Cell Cycle, 2004, 3: 529-533

60 den Elzen N R, O'Connell M J. Recovery from DNA damage checkpoint arrest by PP1-mediated inhibition of Chk1. EMBO J, 2004, 23: 908-918

61 Hsu L C. Identification and functional characterization of a PP1binding site in BRCA1. Biochem Biophys Res Commun, 2007, 360: 507-512

62 Winter S L, Bosnoyan-Collins L, Pinnaduwage D, et al. The interaction of PP1 with BRCA1 and analysis of their expression in breast tumors. BMC Cancer, 2007, 7: 85

$63 \mathrm{Yu}$ Y M, Pace S M, Allen S R, et al. A PP1-binding motif present in BRCA1 plays a role in its DNA repair function. Int J Biol Sci, 2008, 4: 352-361

64 Haneda M, Kojima E, Nishikimi A, et al. Protein phosphatase 1, but not protein phosphatase 2A, dephosphorylates DNA-damaging stressinduced phospho-serine 15 of p53. FEBS Lett, 2004, 567: 171-174

65 Shieh S Y, Ikeda M, Taya Y, et al. DNA damage-induced phosphorylation of p53 alleviates inhibition by MDM2. Cell, 1997, 91: 325334

66 Tibbetts R S, Brumbaugh K M, Williams J M, et al. A role for ATR in the DNA damage-induced phosphorylation of p53. Genes Dev, 1999, 13: 152-157

67 Shieh S Y, Taya Y, Prives C. DNA damage-inducible phosphorylation of p53 at N-terminal sites including a novel site, Ser20, requires tetramerization. EMBO J, 1999, 18: 1815-1823

68 Hirao A, Kong Y Y, Matsuoka S, et al. DNA damage-induced activation of p53 by the checkpoint kinase Chk2. Science, 2000, 287: $1824-1827$

69 Lee S J, Lim C J, Min J K, et al. Protein phosphatase 1 nuclear targeting subunit is a hypoxia inducible gene: Its role in post-translational modification of p53 and MDM2. Cell Death Differ, 2007, 14: 1106-1116

70 Eichhorn P J, Creyghton M P, Bernards R. Protein phosphatase 2A regulatory subunits and cancer. Biochim Biophys Acta, 2009, 1795: $1-15$

71 Inui S, Sanjo H, Maeda K, et al. Ig receptor binding protein 1 (alpha4) is associated with a rapamycin-sensitive signal transduction in lymphocytes through direct binding to the catalytic subunit of protein phosphatase 2A. Blood, 1998, 92: 539-546

72 Bosch M, Cayla X, van Hoof C, et al. The PR55 and PR65 subunits of protein phosphatase 2A from Xenopus laevis. Molecular cloning and developmental regulation of expression. Eur J Biochem, 1995, 230: $1037-1045$

73 Shi Y. Assembly and structure of protein phosphatase 2A. Sci China C-Life Sci, 2009, 52: 135-146

74 Guenin S, Schwartz L, Morvan D, et al. PP2A activity is controlled by methylation and regulates oncoprotein expression in melanoma cells: A mechanism which participates in growth inhibition induced by chloroethylnitrosourea treatment. Int J Oncol, 2008, 32: 49-57

75 Avni D, Yang H, Martelli F, et al. Active localization of the retinoblastoma protein in chromatin and its response to S phase DNA damage. Mol Cell, 2003, 12: 735-746

76 Dohoney K M, Guillerm C, Whiteford C, et al. Phosphorylation of p53 at serine 37 is important for transcriptional activity and regulation in response to DNA damage. Oncogene, 2004, 23: 49-57

77 Li H H, Cai X, Shouse G P, et al. A specific PP2A regulatory subunit, B56gamma, mediates DNA damage-induced dephosphorylation of p53 at Thr55. EMBO J, 2007, 26: 402-411

78 Yan Y, Cao P T, Greer P M, et al. Protein phosphatase 2A has an essential role in the activation of gamma-irradiation-induced G2/M checkpoint response. Oncogene, 2010, 29: 4317-4329

79 Jang Y J, Ji J H, Choi Y C, et al. Regulation of Polo-like kinase 1 by DNA damage in mitosis: Inhibition of mitotic PLK-1 by protein phosphatase 2A. J Biol Chem, 2007, 282: 2473-2482

80 Margolis S S, Perry J A, Forester C M, et al. Role for the PP2A/ B56delta phosphatase in regulating 14-3-3 release from Cdc25 to control mitosis. Cell, 2006, 127: 759-773

81 Douglas P, Moorhead G B, Ye R, et al. Protein phosphatases regulate DNA-dependent protein kinase activity. J Biol Chem, 2001, 276: 18992-18998

82 McConnell J L, Gomez R J, McCorvey L R, et al. Identification of a PP2A-interacting protein that functions as a negative regulator of phosphatase activity in the ATM/ATR signaling pathway. Oncogene, 2007, 26: 6021-6030

83 Chowdhury D, Xu X, Zhong X, et al. A PP4-phosphatase complex dephosphorylates gamma-H2AX generated during DNA replication. Mol Cell, 2008, 31: 33-46

84 O’Neill B M, Szyjka S J, Lis E T, et al. Pph3-Psy2 is a phosphatase complex required for Rad53 dephosphorylation and replication fork restart during recovery from DNA damage. Proc Natl Acad Sci USA, 2007, 104: 9290-9295

85 Veluthakal R, Wadzinski B E, Kowluru A. Localization of a nuclear serine/threonine protein phosphatase in insulin-secreting INS-1 cells: Potential regulation by IL-1beta. Apoptosis, 2006, 11: 1401-1411

86 Sumiyoshi E, Sugimoto A, Yamamoto M. Protein phosphatase 4 is required for centrosome maturation in mitosis and sperm meiosis in $C$. elegans. J Cell Sci, 2002, 115: 1403-1410

87 Lyu J, Jho E H, Lu W. Smek promotes histone deacetylation to suppress transcription of Wnt target gene brachyury in pluripotent embryonic stem cells. Cell Res, 2011, 21: 911-921

88 Gingras A C, Caballero M, Zarske M, et al. A novel, evolutionarily conserved protein phosphatase complex involved in cisplatin sensitivity. Mol Cell Proteomics, 2005, 4: 1725-1740

89 Kim J A, Hicks W M, Li J, et al. Protein phosphatases pph3, ptc2, and ptc3 play redundant roles in DNA double-strand break repair by homologous recombination. Mol Cell Biol, 2011, 31: 507-516

90 Nussenzweig A, Paull T. DNA repair: Tails of histones lost. Nature, 2006, 439: 406-407

91 Szyjka S J, Aparicio J G, Viggiani C J, et al. Rad53 regulates replication fork restart after DNA damage in Saccharomyces cerevisiae. Genes Dev, 2008, 22: 1906-1920

92 Nakada S, Chen G I, Gingras A C, et al. PP4 is a gamma H2AX phosphatase required for recovery from the DNA damage checkpoint. EMBO Rep, 2008, 9: 1019-1026

93 Lee D H, Pan Y, Kanner S, et al. A PP4 phosphatase complex dephosphorylates RPA2 to facilitate DNA repair via homologous recombination. Nat Struct Mol Biol, 2010, 17: 365-372

94 Wang B, Zhao A, Sun L, et al. Protein phosphatase PP4 is overexpressed in human breast and lung tumors. Cell Res, 2008, 18: 974977

95 Bastians $\mathrm{H}$, Ponstingl $\mathrm{H}$. The novel human protein serine/threonine phosphatase 6 is a functional homologue of budding yeast Sit $4 p$ and fission yeast ppe1, which are involved in cell cycle regulation. J Cell Sci, 1996, 109(Pt12): 2865-2874

96 Stefansson B, Brautigan D L. Protein phosphatase 6 subunit with conserved Sit4-associated protein domain targets IkappaBepsilon. J Biol Chem, 2006, 281: 22624-22634

97 Stefansson B, Ohama T, Daugherty A E, et al. Protein phosphatase 6 regulatory subunits composed of ankyrin repeat domains. Biochemistry, 2008, 47: 1442-1451 
98 Guergnon J, Derewenda U, Edelson J R, et al. Mapping of protein phosphatase- 6 association with its SAPS domain regulatory subunit using a model of helical repeats. BMC Biochem, 2009, 10: 24

99 Douglas P, Zhong J, Ye R, et al. Protein phosphatase 6 interacts with the DNA-dependent protein kinase catalytic subunit and dephosphorylates gamma-H2AX. Mol Cell Biol, 2010, 30: 1368-1381

100 Mi J, Dziegielewski J, Bolesta E, et al. Activation of DNA-PK by ionizing radiation is mediated by protein phosphatase 6 . PLoS One, 2009, 4: e4395

101 Douglas P, Moorhead G, Xu X, et al. Choreographing the DNA damage response: PP6 joins the dance. Cell Cycle, 2010, 9: 12211222

102 Zhong J, Liao J, Liu X, et al. Protein phosphatase PP6 is required for homology-directed repair of DNA double-strand breaks. Cell Cycle, 2011, 10: 1411-1419

103 Hinds T D Jr, Sanchez E R. Protein phosphatase 5. Int J Biochem Cell Biol, 2008, 40: 2358-2362

104 Ham B M, Jayachandran H, Yang F, et al. Novel Ser/Thr protein phosphatase 5 (PP5) regulated targets during DNA damage identified by proteomics analysis. J Proteome Res, 2010, 9: 945-953

105 Zhang J, Bao S, Furumai R, et al. Protein phosphatase 5 is required for ATR-mediated checkpoint activation. Mol Cell Biol, 2005, 25: 9910-9919

106 Ali A, Zhang J, Bao S, et al. Requirement of protein phosphatase 5 in DNA-damage-induced ATM activation. Genes Dev, 2004, 18: 249-254

107 Kang Y, Cheong H M, Lee J H, et al. Protein phosphatase 5 is necessary for ATR-mediated DNA repair. Biochem Biophys Res Commun, 2011, 404: 476-481

108 Yong W, Bao S, Chen H, et al. Mice lacking protein phosphatase 5 are defective in ataxia telangiectasia mutated (ATM)-mediated cell cycle arrest. J Biol Chem, 2007, 282: 14690-14694

109 Kang Y, Lee J H, Hoan N N, et al. Protein phosphatase 5 regulates the function of 53BP1 after neocarzinostatin-induced DNA damage. J Biol Chem, 2009, 284: 9845-9853

110 Golden T, Aragon I V, Rutland B, et al. Elevated levels of Ser/Thr protein phosphatase 5 (PP5) in human breast cancer. Biochim Biophys Acta, 2008, 1782: 259-270

111 Fiscella M, Zhang H, Fan S, et al. Wip1, a novel human protein phosphatase that is induced in response to ionizing radiation in a p53- dependent manner. Proc Natl Acad Sci USA, 1997, 94: 60486053

112 Takekawa M, Adachi M, Nakahata A, et al. p53-inducible wip1 phosphatase mediates a negative feedback regulation of p38 MAPKp53 signaling in response to UV radiation. EMBO J, 2000, 19: 6517-6526

113 Yamaguchi H, Minopoli G, Demidov O N, et al. Substrate specificity of the human protein phosphatase 2Cdelta, Wip1. Biochemistry, 2005, 44: 5285-5294

114 Wang A, Gu J, Judson-Kremer K, et al. Response of human mammary epithelial cells to DNA damage induced by BPDE: Involvement of novel regulatory pathways. Carcinogenesis, 2003, 24: 225-234

115 Harrison M, Li J, Degenhardt Y, et al. Wip1-deficient mice are resistant to common cancer genes. Trends Mol Med, 2004, 10: 359-361

116 Castellino R C, De Bortoli M, Lu X, et al. Medulloblastomas over- express the p53-inactivating oncogene WIP1/PPM1D. J Neurooncol, 2008, 86: 245-256

117 Le Guezennec X, Bulavin D V. WIP1 phosphatase at the crossroads of cancer and aging. Trends Biochem Sci, 2010, 35: 109-114

118 Lu X, Nguyen T A, Moon S H, et al. The type 2C phosphatase Wip1: An oncogenic regulator of tumor suppressor and DNA damage response pathways. Cancer Metastasis Rev, 2008, 27: 123-135

119 Lindqvist A, de Bruijn M, Macurek L, et al. Wip1 confers G2 checkpoint recovery competence by counteracting p53-dependent transcriptional repression. EMBO J, 2009, 28: 3196-3206

120 Lu X, Nannenga B, Donehower L A. PPM1D dephosphorylates Chk1 and p53 and abrogates cell cycle checkpoints. Genes Dev, 2005, 19: 1162-1174

121 Yamaguchi H, Durell S R, Chatterjee D K, et al. The Wip1 phosphatase PPM1D dephosphorylates SQ/TQ motifs in checkpoint substrates phosphorylated by PI3K-like kinases. Biochemistry, 2007, 46: 12594-12603

122 Lu X, Ma O, Nguyen T A, et al. The Wip1 phosphatase acts as a gatekeeper in the p53-Mdm2 autoregulatory loop. Cancer Cell, 2007, 12: 342-354

123 Lu X, Bocangel D, Nannenga B, et al. The p53-induced oncogenic phosphatase PPM1D interacts with uracil DNA glycosylase and suppresses base excision repair. Mol Cell, 2004, 15: 621-634

124 Craig A L, Chrystal J A, Fraser J A, et al. The MDM2 ubiquitination signal in the DNA-binding domain of p53 forms a docking site for calcium calmodulin kinase superfamily members. Mol Cell Biol, 2007, 27: 3542-3555

125 Lee J H, Paull T T. ATM activation by DNA double-strand breaks through the Mre11-Rad50-Nbs1 complex. Science, 2005, 308: 551554

126 Guo J Y, Yamada A, Kajino T, et al. Aven-dependent activation of ATM following DNA damage. Curr Biol, 2008, 18: 933-942

127 Lu X, Nguyen T A, Donehower L A. Reversal of the ATM/ATRmediated DNA damage response by the oncogenic phosphatase PPM1D. Cell Cycle, 2005, 4: 1060-1064

128 Cha H, Lowe J M, Li H, et al. Wip1 directly dephosphorylates gamma-H2AX and attenuates the DNA damage response. Cancer Res, 2010, 70: 4112-4122

129 Macurek L, Lindqvist A, Voets O, et al. Wip1 phosphatase is associated with chromatin and dephosphorylates gammaH2AX to promote checkpoint inhibition. Oncogene, 2010, 29: 2281-2291

130 Rogakou E P, Pilch D R, Orr A H, et al. DNA double-stranded breaks induce histone H2AX phosphorylation on serine 139. J Biol Chem, 1998, 273: 5858-5868

131 Paull T T, Rogakou E P, Yamazaki V, et al. A critical role for histone $\mathrm{H} 2 \mathrm{AX}$ in recruitment of repair factors to nuclear foci after DNA damage. Curr Biol, 2000, 10: 886-895

132 Rogakou E P, Boon C, Redon C, et al. Megabase chromatin domains involved in DNA double-strand breaks in vivo. J Cell Biol, 1999, 146: 905-916

133 Chowdhury D, Keogh M C, Ishii H, et al. gamma-H2AX dephosphorylation by protein phosphatase 2A facilitates DNA double-strand break repair. Mol Cell, 2005, 20: 801-809

134 Kimura H, Takizawa N, Allemand E, et al. A novel histone exchange factor, protein phosphatase $2 \mathrm{Cgamma}$, mediates the exchange and dephosphorylation of H2A-H2B. J Cell Biol, 2006, 175: 389-400

Open Access This article is distributed under the terms of the Creative Commons Attribution License which permits any use, distribution, and reproduction in any medium, provided the original author(s) and source are credited. 